\title{
Análise eletromiográfica de músculos do tronco e pelve durante o leg circle no solo e com molas no Cadillac
}

\author{
Electromyographic analysis of trunk and pelvis muscles during Leg Circle on the mat and \\ with springs on the Cadillac
}
Análisis electromiográfico de los músculos del torso y la pelvis durante el círculo con una pierna en el suelo y con muelles en Cadillac

Rosangela Menezes de Paula', William Dhein², Catiane Souza³, Edgar Santiago Wagner Neto ${ }^{4}$, Jefferson Fagundes Loss ${ }^{5}$

RESUMO I O objetivo do estudo é investigar, através da EMG, se é possível alterar a exigência dos músculos estabilizadores do tronco e da pelve, executando o exercício Leg Circle no solo e com diferentes posicionamentos das molas no Cadillac. Treze instrutoras praticantes de Pilates por no mínimo seis meses e sem dor realizaram 10 repetições do exercício Leg Circle em três situações: (1) Solo; (2) Molas altas (MA); (3) Molas médias (MM). Foram obtidos dados de EMG dos músculos oblíquo interno do abdômen/transverso do abdômen (OI/TS), oblíquo externo do abdômen (OE), reto abdominal (RA) e multífidos (MU) apresentados em percentual da CIVM. As comparações foram realizadas através da ANOVA de medidas repetidas $(\alpha=0,05)$. Todos os músculos sofreram influência do posicionamento da mola. No solo, as maiores ativações ocorreram nos músculos RA e OE, enquanto nas execuções realizadas com molas, os músculos mais recrutados foram OI/TS (MM) e MU (MA). Os músculos RA e OE não obtiveram diferenças entre as situações MA e MM. A execução do exercício Leg Circle no solo e com molas gera alterações no recrutamento dos músculos avaliados. Observa-se também que, ao analisar o exercício realizado no Cadillac entre molas altas e molas médias, houve diferença apenas para os músculos OI/TS e MU.

Descritores | Técnicas de Exercício e de Movimento; Reabilitação; Eletromiografia.

\begin{abstract}
I This study aimed to investigate, through EMG, whether it is possible to change the requirement of the stabilizing muscles of the trunk and pelvis, performing the Leg Circle exercise on the mat and with different positions of springs on the Cadillac. Thirteen Pilates instructors practicing for at least six months and without pain performed 10 repetitions of the Leg Circle exercise in three situations: (1) Mat; (2) High springs (HS); (3) Medium springs (MS). EMG data were obtained from the abdominal internal oblique/transverse abdominal (IO/TVA), abdominal external oblique (EO), rectus abdominis (RA), and multifidus (MU) muscles, presented as a percentage of Maximum Voluntary Isometric Contraction (MVIC). Comparisons were carried out using repeated measures ANOVA ( $\alpha=0.05)$. All muscles were influenced by the positioning of the spring. On the mat, the greatest activations occurred in the RA and EO muscles, while in the executions performed with springs,
\end{abstract}


the most recruited muscles were IO/TVA (MS) and MU (HS). RA and OE muscles did not show differences between the HS and MS situations. The execution of the Leg Circle exercise on the mat and with springs generates changes in the recruitment of the evaluated muscles. We also observed that, when analyzing the exercise performed on the Cadillac between high and medium springs, there was a difference only for the IO/TVA and MU muscles. Keywords | Exercise and Movement Techniques; Rehabilitation; Electromyography.

RESUMEN | El objetivo del estudio el investigar, através de la EMG, si es posible alterar la exigencia de los músculos estabilizadores del tronco y de la pelvis, ejecutando el ejercicio Leg Circle en el suelo y con diferentes posicionamientos de los muelles en el Cadillac. Trece instructores profesionales de Pilates durante al menos seis meses y sin dolor realizó 10 repeticiones del ejercicio Leg Circle en tres situaciones: (1) El suelo; (2) Muelles altos (MA);
(3) Muelles medianos (MM). Se obtuvieron datos de EMG de los músculos oblicuos internos del abdomen / transverso del abdomen (OI / TS), oblicuo externo del abdomen (OE), recto abdominal (RA) y multífidos (MU) presentados en porcentaje de la CIVM. Las comparaciones se realizaron a través de la ANOVA de medidas repetidas $(\alpha=0,05)$. Todos los músculos sufrieron influencia del posicionamiento del muelle. En el suelo, las mayores activaciones ocurrieron en los músculos RA y OE, mientras que en las ejecuciones realizadas con muelles, los músculos más reclutados fueron OI / TS (MM) y MU (MA). Los músculos RA y OE no obtuvieron diferencias entre las situaciones MA y MM. La ejecución del ejercicio Leg Circle en el suelo y con muelles genera cambios en el reclutamiento de los músculos evaluados. Se observa también que, al analizar el ejercicio realizado en el Cadillac entre muelles altos y muelles medianos, hubo diferencia sólo para los músculos OI / TS y MU. Palabras clave I Técnicas de Ejercicio y de Movimiento; Rehabilitación; Electromiografía.

\section{INTRODUÇÃO}

O método pilates tornou-se popular como atividade física e como ferramenta de reabilitação, buscando integrar a mente e o corpo dos praticantes ${ }^{1-3}$. Manter a estabilidade do tronco e da pelve, durante a realização dos exercícios, é uma das principais características deste método ${ }^{1,4}$.

Enquanto programas de treinamentos clássicos como o treinamento de força, utilizam o incremento de cargas para progressão do treino ${ }^{5}$, o método pilates aumenta os desafios de instabilidade, por meio de variações na execução dos exercícios ou mesmo nas cargas oriundas das molas à medida que o praticante vai evoluindo no treinamento ${ }^{6}$. Não obstante, o incremento da progressão dentro do método tem sido feito de forma subjetiva ${ }^{7}$.

Um dos exercícios frequentemente utilizados com o objetivo de desafiar a estabilidade do quadril/pelve, além de promover a integridade funcional e anatômica da articulação do quadril é o Leg Circle ou Hip Circle. Este exercício pode ser executado tanto no solo, sem qualquer acessório, ou no Cadillac, onde o indivíduo é posicionado em decúbito dorsal e as molas são colocadas na cabeceira do aparelho e nos membros inferiores, na região dos pés ${ }^{6,8}$.

A altura de fixação, diferentes constantes de deformação ou ausência das molas, durante o exercício, tem como objetivo auxiliar ou dificultar a manutenção desta estabilidade. Já foi mostrado em outros exercícios que diferentes posicionamentos de molas alteram as respostas musculares ${ }^{6,7,9}$. No entanto, até onde pode ser investigado, não há informações referentes aos efeitos de diferentes posicionamentos das molas na estabilidade do exercício Leg Circle. Sendo assim, o presente estudo tem como objetivo investigar, através da eletromiografia de superfície (EMG), se é possível alterar a exigência dos músculos estabilizadores do tronco e da pelve, executando o exercício Leg Circle no solo e com diferentes posicionamentos das molas no Cadillac.

\section{METODOLOGIA}

\section{Amostra}

A estimativa amostral foi realizada no software G*Power 3.1.9.2, adotando os seguintes critérios: tamanho de efeito de 0,40 ; probabilidade de erro de $5 \%$; poder estatístico de 80\%; correlação entre as medições de 0,5; assumindo esfericidade $(\mathrm{E}=1)$; para a família de testes estatísticos ANOVA para medidas repetidas. $\mathrm{O}$ cálculo definiu o tamanho amostral em 10 sujeitos. Em função de possíveis perdas amostrais, foram avaliadas 13 instrutoras de Pilates. As participantes apresentavam idade média de 31,3 anos $( \pm 5,3)$, altura de $1,61 \mathrm{~cm}( \pm 0,05)$ e peso de $61,2 \mathrm{~kg}( \pm 6,1)$. As participantes foram selecionadas de forma intencional, onde foram adotados os seguintes 
critérios de elegibilidade: ser instrutora de Pilates a, no mínimo, seis meses; estar praticando ao menos uma vez por semana a, no mínimo, seis meses; não apresentar dor lombar aguda ou crônica, dor nos membros inferiores ou na região abdominal.

\section{Procedimentos}

Todas as coletas foram realizadas no Laboratório de Pesquisa do Exercício da Universidade Federal do Rio Grande do Sul, agendadas conforme disponibilidade das participantes. Inicialmente, as participantes assinaram o Termo de Consentimento Livre e Esclarecido, onde constaram os objetivos e procedimentos do estudo. Para cada amostra foi realizada uma avaliação inicial onde foram obtidos dados de peso, altura e idade. Seguido por um aquecimento, alongamentos e familiarização com os exercícios propostos. Após esta etapa inicial a participante foi preparada para a coleta de dados.

Inicialmente, foram realizadas a tricotomia e limpeza da pele com álcool nos músculos avaliados. Foram fixados eletrodos de superfície descartáveis (Miotec DoubleTrace - 100; Ag/ $\mathrm{AgCl}, 10 \mathrm{~mm}$ de diâmetro, autoadesiva, na configuração bipolar), unilateralmente a direita em músculos do Powerhouse ${ }^{1}$, sendo: Reto Abdominal (RA), Oblíquo Externo do Abdômen (OE), Oblíquo Interno do Abdômen/Transverso do Abdômen (OI/TS) e Multífidos (MU). Após a colocação dos eletrodos, foram realizados os testes de contração isométrica voluntária máxima (CIVM) de cada músculo. As CIVMs de cada um dos músculos tiveram duração de 5 segundos com incentivo verbal e realizadas duas repetições com intervalo de dois minutos. O posicionamento dos eletrodos e a realização das CIVM seguiram recomendações da literatura $^{9-11}$. A aquisição dos dados de eletromiografia foi obtida utilizando o eletromiógrafo da marca BTS FREEEMG 1000 com uma taxa de amostragem de $1000 \mathrm{~Hz}$. O software utilizado para aquisição dos dados eletromiográficos foi o BTS Smart Capture.

Após as CIVMs, foram fixados bilateralmente marcadores reflexivos nos seguintes acidentes ósseos: Espinha Ilíaca anterossuperior, Trocanter Maior, Côndilo Lateral Femoral e Maléolo Lateral. Os dados cinemáticos foram obtidos através do sistema BTS SMARTDX 700, composto por 10 câmeras infravermelhas com taxa de amostragem de $100 \mathrm{~Hz}$ e foram utilizados para recortes e análises dos sinais EMG. As participantes foram posicionadas no Cadillac (Physio Pilates ${ }^{\circledR}$ ) em decúbito dorsal, centralizadas, com a cabeça próxima a torre onde se encontram as molas. As participantes executaram o movimento de $\mathrm{Leg}$ Circle em três situações (Figura 1): (1) Leg Circle no Solo; (2) Leg Circle com molas altas (MA); (3) Leg Circle com molas médias (MM). Em MA, o quadril inicia com flexão de $90^{\circ}$, enquanto em MM o quadril inicia em $45^{\circ}$. Os membros inferiores estão relaxados, mantendo a posição neutra, caracterizada por um equilíbrio de torques entre as molas e o peso do segmento. A instrutora experiente orientou quanto à posição neutra da pelve. Foram realizadas dez repetições dos gestos numa velocidade de execução comumente realizada pela participante em sentido lateral para medial. Todas as execuções foram randomizadas.
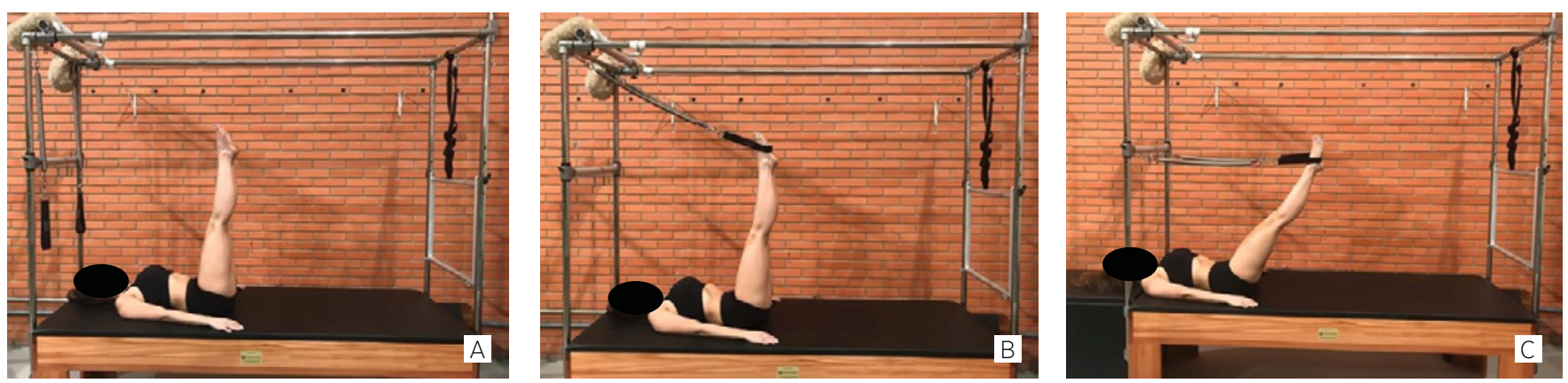

Figura 1. Exercício leg circle em diferentes situações

A: leg circle solo; B: leg circle com molas altas; C: leg circle com molas médias.

\section{Análise dos dados}

A análise dos dados eletromiográficos foi realizada no software BTS Smart Analyzer onde os sinais coletados foram submetidos a um procedimento de filtragem utilizando um filtro Butterworth passa banda de $4^{\circ}$ ordem, com banda de frequência entre 20 e $400 \mathrm{~Hz}$. Após o procedimento de filtragem do sinal eletromiográfico foram 
realizados os recortes das repetições, utilizando como base a cinemática do membro inferior. Foram calculados os valores RMS (root mean square) e normalizados a partir do valor de pico do envelope RMS obtido na CIVM.

A análise estatística for realizada no software SPSS 20.0. As comparações dos dados eletromiográficos para os músculos do Powerhouse foram realizadas através da ANOVA de medidas repetidas sendo consideradas as situações: (1) Solo, (2) Molas Altas e (3) Molas Médias. Em caso de diferenças estatísticas nas comparações foi utilizado o teste de post hoc de Bonferroni. O nível de significância adotado foi de $\alpha<0,05$.

\section{RESULTADOS}

Os dados da atividade EMG em percentual da CIVM para cada um dos músculos estudados e nos diferentes posicionamentos podem ser visualizados na Figura 2. Com base nos resultados da ANOVA de medidas repetidas, pode-se observar que o posicionamento ou ausência das molas afeta a ativação EMG dos músculos: $\mathrm{RA}\left[\mathrm{F}(2 ; 24)=41,383 ; \mathrm{p}=<0,001 ; \eta^{2}=0,775\right] ; \mathrm{OE}[\mathrm{F}(1,062$; $\left.12,743)=22,996 ; \mathrm{p}=<0,001 ; \eta^{2}=0,657\right] ; \mathrm{OI} / \mathrm{TS}[\mathrm{F}(1,246$; $\left.14,948)=13,184 ; \mathrm{p}=0,002 ; \eta^{2}=0,524\right] ; \mathrm{MU}[\mathrm{F}(1,271$; $\left.15,251)=8,223 ; \mathrm{p}=0,008 ; \eta^{2}=0,407\right]$.

Os músculos RA e $\mathrm{OE}$ obtiveram as maiores atividades EMG efetuados no solo quando comparadas com aquelas executadas com as molas na posição alta $(p=<0,001)$ e média $(p=<0,001)$. Não houve diferença quando comparados às situações de mola alta com mola média para ambos os músculos $(\mathrm{p}=1)$.

O músculo OI/TS obteve as maiores atividades EMG no solo e mola média, não ocorrendo diferença entre estas situações $(p=0,072)$. Houve diferença entre a situação solo e mola alta $(p=<0,001)$ e entre as molas $(p=0,02)$. O músculo MU obteve as maiores atividades EMG na situação de mola alta quando comparado a mola média ( $\mathrm{p}=0,01)$. Não houve diferença quando comparados às situações solo com mola alta $(\mathrm{p}=0,075)$ ou mola média $(\mathrm{p}=1)$.

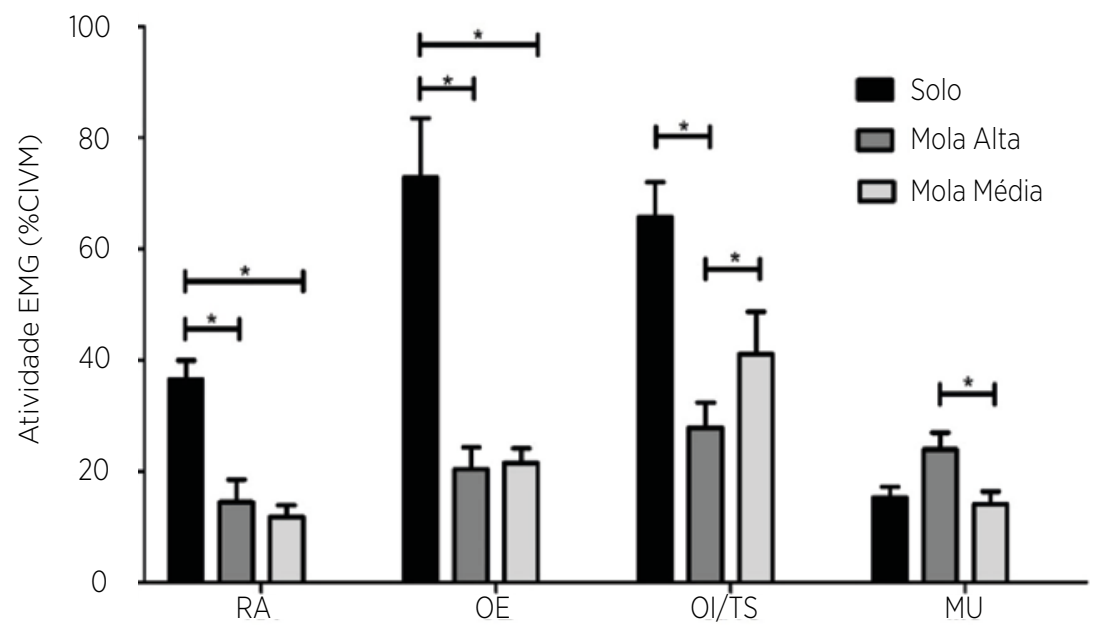

Figura 2. Média e erro-padrão da ativação EMG em percentual da CIVM durante o leg circle no solo, com mola alta e média 'Diferença significativa: p<0,05; RA: reto abdominal; OE: oblíquo externo do abdômen; OI/TS: oblíquo interno do abdômen/transverso do abdômen; MU: multífidos.

\section{DISCUSSÃO}

O proposto artigo tem como objetivo investigar, através da eletromiografia de superfície, se é possível alterar a exigência dos músculos estabilizadores do tronco e da pelve, executando o exercício Leg Circle no solo e com diferentes posicionamentos das molas no Cadillac. Pôdese constatar que os músculos RA, OE, OI/TS e MU sofreram influência da mudança de posicionamento ou ausência das molas na execução do exercício de Leg Circle.

Os músculos RA e OE obtiveram suas maiores atividades EMG na situação solo. Esse resultado pode ter ocorrido devido a maior exigência de contração muscular na situação solo, onde não há um suporte externo ${ }^{12,13}$, pois comparado às situações MA e MM, o movimento se torna facilitado pelas molas, independente da altura da ancoragem. Esta facilitação ocorre principalmente durante a fase excêntrica do movimento, onde as molas auxiliam no retorno à posição inicial. Na literatura, o comportamento EMG de músculos do Powerhouse durante diferentes exercícios no solo são avaliados ${ }^{12,14-16}$. Nenhum destes estudos avaliou o exercício de Leg Circle ou Hip Circle, porém, realizaram exercícios com mola e membros inferiores elevados. 
Observou-se o alto recrutamento principalmente dos músculos $\mathrm{RA}^{12}$ e $\mathrm{OE}^{14,15}$, o que concorda com os achados do presente estudo, que também obtiveram altas magnitudes para estes músculos nas situações realizadas no solo.

Em ambos os casos com molas, o baixo recrutamento da atividade elétrica para o RA e OE pode-se justificar pela facilitação que elas oferecem auxiliando no movimento, fazendo com que estes músculos mais superficiais não sejam recrutados, diferentemente dos músculos OI e MU que são mais profundos. Enquanto na situação MA a exigência da cadeia posterior é maior com o recrutamento do MU e músculos que atuam no quadril ${ }^{7}$, na situação $\mathrm{MM}$ essa exigência se torna menor, fazendo que o recrutamento do músculo $\mathrm{MU}$ diminua. Como os músculos RA e OE não alteram sua atividade EMG na situação com suporte externo das molas, o músculo OI aumenta seu recrutamento para compensar a alteração da cadeira posterior, reforçando sua importância na estabilização do complexo lombopélvico $^{13,17,18}$. Na literatura, a comparação entre molas altas e molas baixas já foi verificada ${ }^{9}$ no exercício de extensão e flexão de quadril. $\mathrm{O}$ músculo $\mathrm{OE}$ apresentou maior atividade EMG na situação molas baixas, discordando com o presente estudo, que não obteve diferenças entre a situação MA e MM para este músculo. Neste estudo, esta diferença foi encontrada apenas para o OI, que não foi avaliado no estudo citado 9 . Especulamos que o resultado, possa ser proveniente da função estabilizadora da região lombo-pélvica realizada pelos músculos mais profundos do abdômen, como o obliquo interno ${ }^{4}$, bem como, a posição da mola ser média em nosso estudo e não baixa como na literatura9.

Apesar dos resultados expressivos e sua aplicabilidade, o estudo apresenta algumas limitações, tais como: os músculos que atuam na movimentação do quadril não foram analisados. Essa afirmação pode responder questões que foram especuladas com base de estudos da literatura. Assim, a relação entre Powerhouse e musculatura de quadril pode ser investigada em estudos futuros. Também podemos ressaltar que as amostras, por apresentarem diferentes níveis de força muscular e/ou padronização das molas utilizadas durante o estudo, podem influenciar no modo de execução e resultado. Apesar de não individualizar as resistências exercidas pelas molas, teve-se o cuidado metodológico de padronizar o ângulo de $45^{\circ}(\mathrm{MM})$ e $90^{\circ}$ (MA) da pelve e quadril. $\mathrm{Na}$ situação $\mathrm{MA}$, existe a dificuldade de execução para algumas amostras devido à resistência exercida pelas molas, podendo gerar compensações.
Quanto à aplicação prática do estudo, os instrutores de Pilates que objetivam prescrever exercícios com maior recrutamento da musculatura mais superficial como RA e $\mathrm{OE}$, devem tomar como escolha os exercícios realizados no solo, enquanto que, quando objetivar musculatura mais profunda, OI e MU, a escolha deve ser pelos exercícios com mola média e alta.

Dentro do método Pilates existe um vasto repertório de exercícios com inúmeras possibilidades. Alguns exercícios são mais eficazes que outros para trabalhar a estabilidade do tronco e encontrar maior ativação do Powerhouse. Para alcançar os objetivos propostos é necessário que os exercícios sejam realizados de forma efetiva. Com esse trabalho, os profissionais habilitados poderão, no momento da montagem do repertório, fazer a melhor escolha na prescrição dos exercícios, levando em consideração o grau de dificuldade, posição das molas, forma de execução e ativação do Powerhouse.

\section{CONCLUSÃO}

A execução do exercício Leg Circle no solo e no Cadillac gera alterações no recrutamento dos músculos estabilizadores do tronco e pelve avaliados. $\mathrm{Na}$ situação solo, os músculos RA e OE são mais ativados que nas situações com molas. O músculo OI/TS apresenta maiores atividades em solo e com mola média. Já para o músculo MU a maior ativação ocorreu na situação MA. Instrutores que objetivam prescrever exercícios com maior recrutamento da musculatura mais superficial como RA e OE, devem tomar como escolha os exercícios realizados no solo, enquanto que, quando objetivar musculatura mais profunda, OI e MU, a escolha deve ser pelos exercícios com mola média e alta.

\section{REFERÊNCIAS}

1. Muscolino JE, Cipriani S. Pilates and the "powerhouse"-I. J Bodyw Mov Ther. 2004;8(1):15-24. doi: 10.1016/S1360-8592(03)00057-3

2. Bryan M, Hawson S. The benefits of pilates exercise in orthopaedic rehabilitation. Tech Orthop. 2003;18(1):126-9. doi: 10.1097/00013611-200303000-00018

3. Latey P. The pilates method: history and philosophy. J Bodyw Mov Ther. 2001;5(4):275-82. doi: 10.1054/jbmt.2001.0237

4. Barbosa AC, Vieira ER, Silva AF, Coelho AC, Martins FM, Fonseca DS, et al. Pilates experience vs. muscle activation during abdominal drawing-in maneuver. J Bodyw Mov Ther. 2018;22(2):467-70. doi: 10.1016/j.jbmt.2017.05.002 
5. Cadore EL, Brentano MA, Kruel LFM. Efeitos da atividade física na densidade mineral óssea e na remodelação do tecido ósseo. Rev Bras Med Esporte. 2005;11(6):373-9. doi: 10.1590/S1517-86922005000600013

6. Melo MO, Gomes LE, Silva YO, Bonezi A, Loss JF. Análise do torque de resistência e da força muscular resultante durante exercício de extensão de quadril no pilates e suas implicações na prescrição e progressão. Rev Bras Fisioter. 2011;15(1):23-30. doi: 10.1590/S1413-35552011000100006

7. Silva YO, Melo MO, Gomes LE, Bonezi A, Loss JF. Análise da resistência externa e da atividade eletromiográfica do movimento de extensão de quadril realizado segundo o método pilates. Rev Bras Fisioter. 2009;13(1):82-8. doi: 10.1590/S1413-35552009005000010

8. Aparicio E, Pérez J. El auténtico método pilates: el arte del control. Barcelona: Martínez Roca; 2006.

9. Loss JF, Melo MO, Rosa CH, Santos AB, La Torre M, Silva YO. Atividade elétrica dos músculos oblíquos externos e multífidos durante o exercício de flexoextensão do quadril realizado no Cadillac com diferentes regulagens de mola e posições do indivíduo. Rev Bras Fisioter. 2010;14(6):510-7. doi: 10.1590/S1413-35552010000600010

10. Hermens HJ, Freriks B, Disselhorst-Klug C, Rau G. Development of recommendations for SEMG sensors and sensor placement procedures. J Electromyogr Kinesiol. 2000;10(5):361-74. doi: 10.1016/S1050-6411(00)00027-4

11. Konrad P. The ABC of EMG: a practical introduction to kinesiological electromyography. Seattle: Herman \& Wallace; 2005.
12. Dias JM, Oliveira Menacho M, Mazuquin BF, Obara K, Mostagi FQRC, Lima TB, et al. Comparison of the electromyographic activity of the anterior trunk during the execution of two Pilates exercises teaser and longspine - for healthy people. J Electromyogr Kinesiol. 2014;24(5):689-97. doi: 10.1016/j.jelekin.2014.06.005

13. Marques NR, Morcelli MH, Hallal CZ, Gonçalves M. EMG activity of trunk stabilizer muscles during Centering Principle of Pilates Method. J Bodyw Mov Ther. 2013;17(2):185-91. doi: 10.1016/j.jbmt.2012.06.002

14. Pereira IL, Queiroz B, Loss J, Amorim C, Sacco ICN. Trunk muscle EMG during intermediate pilates mat exercises in beginner healthy and chronic low back pain individuals. J Manipulative Physiol Ther. 2017;40(5):350-7. doi: 10.1016/j.jmpt.2017.02.010

15. Silva GB, Morgan MM, Carvalho WRG, Silva E, Freitas WZ, Silva FF, Souza RA. Electromyographic activity of rectus abdominis muscles during dynamic Pilates abdominal exercises. J Bodyw Mov Ther. 2015;19(4):629-35. doi: 10.1016/j.jbmt.2014.11.010

16. Souza EF, Cantergi D, Mendonça A, Kennedy C, Loss JF. Análise eletromiográfica dos músculos reto femoral e reto abdominal durante a execução dos exercícios hundred e teaser do método pilates. Rev Bras Med Esporte. 2012;18(2):105-8.

17. Queiroz BC, Cagliari MF, Amorim CF, Sacco IC. Muscle activation during four pilates core stability exercises in quadruped position. Arch Phys Med Rehabil. 2010;91(1):86-92. doi: 10.1016/j.apmr.2009.09.016

18. Gouveia KMC, Gouveia EC. O músculo transverso abdominal e sua função de estabilização da coluna lombar. Fisioter Mov. 2008;21(3):45-50. 